\title{
Gabapentin for the Treatment of Chronic Pelvic Pain Syndrome in Patients with High Pain Score
}

\author{
Seok Cho, In-Rae Cho \\ Department of Urology, Inje University Ilsan Paik Hospital, Inje University College of Medicine, Goyang, Korea
}

\begin{abstract}
Purpose: The underlying pathogenic mechanisms of chronic pelvic pain syndrome (CPPS) are unclear. A growing body of evidence suggests that the urogenital pain of CPPS may be neuropathic in origin. The objective of this study was to determine if gabapentin can be an effective treatment for the symptoms of CPPS with severe pain.

Materials and Methods: Thirty five males with CPPS (category IIla 25, IIIb 10) and the National Institutes of Health Chronic Prostatitis Symptom Index (NIH-CPSI) total pain score $\geq 9$ in the previous six months were enrolled in this study between October 2010 and December 2011. The dosage of gabapentin was increased from $300 \mathrm{mg} / \mathrm{d}$ to $600 \mathrm{mg} / \mathrm{d}$ during the first four weeks. The primary outcome was evaluated as an improvement in the NIH-CPSI from the baseline to week eight. Results: This study examined 35 males with CPPS and a mean age of 54.2 \pm 9.8 years, mean disease duration of $34.2 \pm 27.7$ months, and a mean prostate volume of $24.9 \pm 5.3 \mathrm{ml}$. The decrease in the total $\mathrm{NIH}$-CPSI pain domain at four weeks was significant with no change observed after eight weeks. Between the category IIIa and IIIb CPPS patients, the change in the total pain domain was not significant. Conclusions: Gabapentin may be effective in some males with CPPS who have a high pain score. More gabapentin may be useless and possibly harmful if gabapentin does not decrease the pain at four weeks.
\end{abstract}

Keywords: Chronic prostatitis with chronic pelvic pain syndrome; Gabapentin; Pain; Prostatitis

Copyright $\odot 2019$, Korean Association of Urogenital Tract Infection and Inflammation. All rights reserved. (c) (1) () This is an open access article distributed under the terms of the Creative Commons Attribution Non-Commercial License (http://creativecommons.org/licenses/by-nc/4.0) which permits unrestricted non-commercial use, distribution, and reproduction in any medium, provided the original work is properly cited.
Received: 26 July, 2019

Revised: 14 August, 2019

Accepted: 21 August, 2019

\section{INTRODUCTION}

Prostatitis is the most common urological disease in young male patients [1]. Chronic prostatitis (CP) presents as urogenital pain, voiding problems, and/or sexual dysfunction [2]. CP/chronic pelvic pain syndrome (CPPS) is more common than chronic bacterial prostatitis, comprising more than 90\% of CP cases [3].

Despite the high prevalence of CP/CPPS, most clinicians face difficulties in its diagnosis and treatment and have significantly more problems managing prostatitis than prostate cancer [4]. Most clinicians want simplified guidelines for the management of prostatitis, but appropriate evidencebased treatment guidelines for CP/CPPS are unavailable.

One of the reasons why it is difficult to establish treatment guidelines is that the pathophysiology of CP/CPPS is not completely understood. On the other hand, CP/CPPS appears to be caused by stimuli, such as infection, urine reflux or trauma in anatomically or genetically susceptible patients, and is associated with local and central neuropathic 
mechanisms involving areas outside the prostate or pelvic area $[5,6]$. CP/CPPS patients with neuropathic pain related to central nervous system sensitization respond to gabapentinoids in other chronic pain conditions, such as diabetic peripheral neuropathy or post-herpetic neuralgia [7].

This study examined the effects of gabapentin on CP/CPPS patients with severe pain that could not be controlled by traditional methods.

\section{MATERIALS AND METHODS}

\section{Study Design}

This was a prospective study design. After obtaining approval from the institutional review board, data were gathered prospectively between October 2010 and December 2011. Written informed consent was obtained from all patients prior to study enrollment. The inclusion criteria were as follows: age $\geq 18$ years, symptoms of discomfort or pain in the pelvic region for the past six months, and National Institutes of Health Chronic Prostatitis Symptom Index (NIH-CPSI) question 4 score $\geq 9$ out of 10 . Patients with white blood cells in the urine or semen ( $>5$ to $9 /$ high power field) were grouped into category IIIa. Patients with no white blood cells in the urine or semen $(<1$ to $4 /$ high powered field) were grouped into category IIIb. Patients with bacteriuria in the urine culture (category II) were excluded. Patients were also excluded if they had chronic renal disease (calculated creatinine clearance $<60 \mathrm{~mL} / \mathrm{min} / 1.73 \mathrm{~m}^{2}$ ), congestive heart failure (New York Heart Association class III or IV), thrombocytopenia $(<100,000 / \mu 1)$, bleeding diathesis, history of alcohol abuse, allergy to any anti-seizure medication, or sensitivity to gabapentinoids. A total of 35 CPPS male patients (category IIIa 25, IIIb 10) were enrolled in this study.

The gabapentin dosage was increased to $300 \mathrm{mg} / \mathrm{d}$ (100 mg orally three times daily) for two weeks, and then to 600 $\mathrm{mg} / \mathrm{d}$ (200 mg orally three times daily) for two weeks if the patient was unsatisfied with the effects. When the appropriate dosage was determined, it was maintained for eight weeks.

The patients completed the NIH-CPSI questionnaire, which contains questions regarding the severity of pain, voiding symptoms, and the quality of life impact at the baseline, and after four and eight weeks of treatment. The primary outcome was defined as a decrease (improvement) in the
NIH-CPSI pain domain $\leq 2$ points from baseline to week eight.

\section{Statistical Analysis}

Statistical analyses were performed using IBM SPSS Statistics version 20.0 (IBM Corp., Armonk, NY, USA), and all reported $\mathrm{p}$-values were two-sided with $\mathrm{p}<0.05$ considered significant. A Mann-Whitney U-test was applied to assess the differences in the continuous variables between the groups. The changes in the measurements over time between the groups were analyzed using the repeated measures analysis of variation and generalized linear modelrepeated measures.

\section{RESULTS}

Twenty five patients (71.4\%) had inflammatory CP/CPPS (category IIIa) and 10 patients (28.6\%) had non-inflammatory CP/CPPS (category IIIb). The mean patient age was $51.3 \pm$ 10.9 years, $58.6 \pm 6.8$ years, and $54.2 \pm 9.8$ in categories IIIa and IIIb and the total patients, respectively. The mean duration of the CPPS symptoms in category IIIa, IIIb groups and total patients was $36.7 \pm 31.5$ months, $30.4 \pm 21.8$ months, and $34.2 \pm 27.7$ months, respectively. The prostate size in category IIIa and IIIb and total patients was $23.8 \pm 5.2 \mathrm{ml}$, $26.6 \pm 5.7 \mathrm{ml}$, and $24.9 \pm 5.3 \mathrm{ml}$, respectively. The mean $\mathrm{NIH}-$ CPSI pain domain in category IIIa and IIIb and total patients was $12.80 \pm 2.43,13.00 \pm 2.20$, and $12.88 \pm 2.36$, respectively. No significant differences were observed between categories IIIa and IIIb with respect to the baseline characteristics. Table 1 lists the baseline patient characteristics.

Table 2 presents the changes in the NIH-CPSI pain domain from the baseline to the four and eight weeks of gabapentin treatment. The NIH-CPSI pain score had improved significantly in categories IIIa and IIIb by week four [-2.33 \pm 3.38 $(p=0.0124)$ and $-2.90 \pm 2.32(p=0.0369)$, respectively]. In the

Table 1. Baseline characteristics of the patients

\begin{tabular}{lccc}
\hline \multirow{2}{*}{ Characteristic } & \multicolumn{3}{c}{ Category of CPPS } \\
\cline { 2 - 4 } & IIla $(\mathrm{n}=25)$ & IIIb $(\mathrm{n}=10)$ & Total $(\mathrm{n}=35)$ \\
\hline Age $(\mathrm{y})$ & $51.3 \pm 10.9$ & $58.6 \pm 6.8$ & $54.2 \pm 9.8$ \\
Duration of symptoms $(\mathrm{mo})$ & $36.7 \pm 31.5$ & $30.4 \pm 21.8$ & $34.2 \pm 27.7$ \\
Prostate volume $(\mathrm{ml})$ & $23.8 \pm 5.2$ & $26.6 \pm 5.7$ & $24.9 \pm 5.3$ \\
Sum of pain domain & $12.80 \pm 2.43$ & $13.00 \pm 2.20$ & $12.88 \pm 2.36$ \\
\hline
\end{tabular}

Values are presented as mean \pm standard deviation.

CPPS: chronic pelvic pain syndrome. 
Table 2. Outcome measurements based on the NIH-CPSI pain domain at baseline, four weeks and four weeks

\begin{tabular}{|c|c|c|c|c|c|c|}
\hline \multirow{2}{*}{ Category of CPPS } & \multicolumn{6}{|c|}{ Sum of pain domain (differences from baseline) } \\
\hline & Baseline & At 4 weeks & $p$-value ${ }^{a)}$ & At 8 weeks & $p$-value $e^{\text {b) }}$ & $p$-value ${ }^{c}$ \\
\hline IIIa $(n=25)$ & $12.80 \pm 2.43$ & $10.47 \pm 3.77(2.33 \pm 3.38)$ & 0.0124 & $10.07 \pm 3.65(2.73 \pm 3.22)$ & 0.0031 & 0.7048 \\
\hline IIIb $(n=10)$ & $13.00 \pm 2.20$ & $10.10 \pm 3.50(2.90 \pm 2.32)$ & 0.0369 & $10.50 \pm 4.30(2.50 \pm 3.40)$ & 0.1190 & 0.8221 \\
\hline Total $(n=35)$ & $12.88 \pm 2.36$ & $10.32 \pm 3.67(2.56 \pm 2.97)$ & 0.0009 & $10.24 \pm 3.93(2.64 \pm 3.31)$ & 0.0011 & 0.9301 \\
\hline
\end{tabular}

Values are presented as mean \pm standard deviation.

NIH-CPSI: National Institutes of Health Chronic Prostatitis Symptom Index, CPPS: chronic pelvic pain syndrome.

${ }^{\text {a) }}$ Difference between baseline and 4 weeks; ${ }^{\text {b) }}$ difference between baseline and 8 weeks; ${ }^{\text {c) }}$ difference between four weeks and four weeks;

${ }^{\mathrm{a}-\mathrm{c})}$ Results from the Mann-Whitney $U$ test.

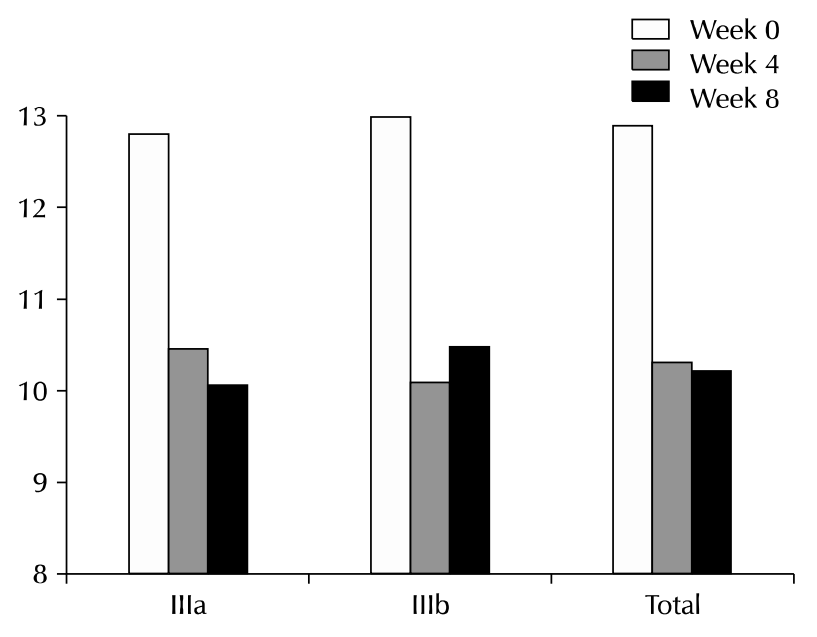

Fig. 1. Pain domain changes from baseline to week 8 in IIIA group, IIIB group and total.

category IIIa patients, the NIH-CPSI pain score improved from the baseline to week eight $(-2.73 \pm 3.22)$, but there was no significant difference from weeks four to eight $(\mathrm{p}=0.7048)$. In the category IIIb patients, the NIH-CPSI pain domain had no significant change from the baseline to week eight $(-2.50 \pm 3.40, p=0.1190)$, even though the changes decreased numerically and there was no significant difference from week four to week eight $(\mathrm{p}=0.8221)$. Between the category IIIa and IIIb CPPS patients, the change in the total pain domain was not significant (repeated measures analysis of variation, generalized linear model-repeated measures). In the total patients, the NIH-CPSI pain domain had decreased significantly at week four $(-2.56 \pm 2.97, \mathrm{p}=0.0009)$ and week eight $(-2.64 \pm 3.31, \mathrm{p}=0.0011)$ but no change was seen between weeks four and eight $(p=0.9301$, Fig. 1).

Adverse events were reported by $11.4 \%$ of patients (4/35), the most common being mild headache in $8.6 \%$ (3/35). One patient (2.9\%) complained of gastrointestinal disturbance. No serious adverse events were encountered and no patient discontinued treatment because of the adverse events.

\section{DISCUSSION}

The pathogenesis of CP/CPPS is unclear. Pontari et al. [8] reported that a history of other neurological diseases was almost five times higher in CPPS than in the controls. Previous studies have related neurological disease and pain to CP/CPPS. A correlation was observed between the biomarkers, such as nerve growth factor, protein-1 $\alpha$, and CCL3, which supports the theory that many $\mathrm{CP} / \mathrm{CPPS}$ patients have neuropathic pain [9-11]. In addition, patients with CP/CPPS have alterations in the autonomic nervous system $[12,13]$. These studies indicate that CP/CPPS may be a part of neurogenic pain syndrome, but the evidence was not direct. For this reason, studies of the use of gabapentinoids have been conducted mostly in females, and favorable results had been shown in randomized controlled trials [14,15].

In the present study, males with a high NIH-CPSI pain score and refractory $\mathrm{CP} / \mathrm{CPPS}$ with previous pain control underwent an eight-week treatment of gabapentin and showed a significant decrease in the NIH-CPSI pain score. No difference was observed between the category IIIa CP group and category IIIb CP group. These results suggest that the refractory pain of $\mathrm{CP} / \mathrm{CPPS}$ patients may be neuropathic rather than simple pain. On the other hand, patients who did not report a decrease in the pain score after undergoing treatment for four weeks showed an effect even after undergoing treatment for eight weeks. Considering that previous studies on gabapentinoids with other chronic neuropathic pain diseases showed improvements in pain only after a few days of treatment, these results suggest that not all patients with high pain scores had neuropathic pain [16].

In a placebo controlled, randomized controlled trial on 324 males with CP/CPPS, Pontari et al. [17] reported that gabapentin was not significantly superior to the placebo according to a six point decrease in the total score on the 
NIH-CPSI scoring system ( $47.2 \%$ vs. $35.8 \% ; \mathrm{p}=0.07$ ). In the secondary outcomes, however, the NIH-CPSI total score decreased by a mean of 6.6 points in the pregabalin arm and 4.2 points in the placebo arm $(\mathrm{p}=0.01)$. Moreover, the pain sub-scores improved by 3.3 (pregabalin) and 2.2 (placebo) $(\mathrm{p}=0.04)$. The NIH-CPSI measures the three key domains of CP/CPPS: pain (location, frequency, and severity; possible score, 0 to 21), urinary symptoms (irritative and obstructive; possible score, 0 to 10), and impact/quality of life (possible score, 0 to 12 ), for a total possible score of 0 to 43 . When designing this study, the authors enrolled patients with a total score of $\geq 15$, and not just the pain score. Because gabapentinoids are effective in pain control, it was expected that they would not work in patients with higher urinary symptoms than the pain symptoms. This study enrolled patients with a high pain score $\geq 9$ and those treated with gabapentin showed significantly reduced pain scores.

Gabapentin and pregabalin are the first options for the treatment of neuropathic pain and appear to act through similar pathways [18]. On the other hand, the results of studies that compared drugs for patients with chronic neuropathic pain are controversial. In a study on patients with diabetic neuropathy, Devi et al. [19] reported that gabapentin and pregabalin had similar efficacy, but pregabalin decreased the pain faster and improved sleep disorders. Similarly, Toth [20] reported that pregabalin may provide additional pain relief in patients with peripheral neuropathy. In contrast, Clark et al. [21] suggested that gabapentin reduced pain more effectively in patients with chronic cancer pain than pregabalin. In a retrospective study, Agarwal and Elsi Sy [22] compared the efficacy of gabapentin and pregabalin in male CPPS patients and used a visual analog score instead of the NIH-CPSI to assess the treatment effectiveness. They reported that gabapentin was superior to pregabalin in achieving a stronger (at least 50\%) response (67.8\% vs. $40 \%, p=0.0012$ ) and a decreased need for adjuvant amitriptyline. In the present study, pregabalin was not approved at the time of the design of the research, so gabapentin was used.

The present study had several limitations. First, the number of patients included in the study was small. Second, although it was a prospective study, there was no control group and no randomized selection was done because there were fewer patients to target. Third, the accuracy of the results may be impaired because only the NIH-CPSI score was used to assess the treatment effects and there was no wash out period to remove any previously used drugs for pain. Furthermore, the dose of gabapentin used in this study was lower than that used for other pain neuropathies.

The results showing that gabapentin decreases the level of pain in refractory CP/CPPS patients with high pain scores are valuable because there is no standard treatment for the pain caused by CP/CPPS. Nevertheless, further research will be needed to identify the markers that can distinguish neuropathic pain from other types of pain in CP/CPPS patient with high pain scores.

\section{CONCLUSIONS}

A gabapentin treatment for eight weeks may provide relief for some CPPS patients with high pain scores and its effect can be confirmed by administration for first four weeks. Based on the present study, the pain of some CPPS patients with high pain scores can be considered as neuropathic pain. On the other hand, if the pain does not decrease at four weeks, it may not be caused by neuropathic pain, so additional gabapentin treatment may be useless because it will only increase the side effects.

\section{CONFLICT OF INTEREST}

No potential conflict of interest relevant to this article was reported.

\section{AUTHOR CONTRIBUTIONS}

I.R.C. and S.C. participated in data collection and wrote the manuscript. I.R.C. participated in the study design. S.C. participated in performing the statistical analysis. I.R.C. and S.C. participated in the study design and coordination and helped to draft the manuscript. All authors read and approved the final manuscript.

\section{ACKNOWLEDGMENTS}

This work was supported by a Grant from Inje University, 2011. 


\section{ORCID}

Seok Cho, https://orcid.org/0000-0001-9404-0502

In-Rae Cho, https://orcid.org/0000-0002-6331-5806

\section{REFERENCES}

1. Collins MM, Stafford RS, O'Leary MP, Barry MJ. How common is prostatitis? A national survey of physician visits. J Urol 1998; 159:1224-8.

2. Rees J, Abrahams M, Doble A, Cooper A; Prostatitis Expert Reference Group (PERG). Diagnosis and treatment of chronic bacterial prostatitis and chronic prostatitis/chronic pelvic pain syndrome: a consensus guideline. BJU Int 2015;116:509-25.

3. Nickel JC, Shoskes D, Wang Y, Alexander RB, Fowler JE Jr, Zeitlin $\mathrm{S}$, et al. How does the pre-massage and post-massage 2-glass test compare to the Meares-Stamey 4-glass test in men with chronic prostatitis/chronic pelvic pain syndrome? J Urol 2006; 176:119-24.

4. Nickel JC, Nigro M, Valiquette L, Anderson P, Patrick A, Mahoney J, et al. Diagnosis and treatment of prostatitis in Canada. Urology 1998;52:797-802.

5. Pontari MA, Ruggieri MR. Mechanisms in prostatitis/chronic pelvic pain syndrome. J Urol 2004;172:839-45.

6. Woodworth D, Mayer E, Leu K, Ashe-McNalley C, Naliboff BD, Labus JS, et al. Unique microstructural changes in the brain associated with urological chronic pelvic pain syndrome (UCPPS) revealed by diffusion tensor MRI, super-resolution track density imaging, and statistical parameter mapping: a MAPP network neuroimaging study. PLoS One 2015;10: e0140250.

7. Moore RA, Wiffen PJ, Derry S, Toelle T, Rice AS. Gabapentin for chronic neuropathic pain and fibromyalgia in adults. Cochrane Database Syst Rev 2014:CD007938.

8. Pontari MA, McNaughton-Collins M, O'leary MP, Calhoun EA, Jang T, Kusek JW, et al. A case-control study of risk factors in men with chronic pelvic pain syndrome. BJU Int 2005;96:55965.

9. Miller LJ, Fischer KA, Goralnick SJ, Litt M, Burleson JA, Albertsen $P$, et al. Nerve growth factor and chronic prostatitis/chronic pelvic pain syndrome. Urology 2002;59:603-8.

10. Desireddi NV, Campbell PL, Stern JA, Sobkoviak R, Chuai S, Shahrara $S$, et al. Monocyte chemoattractant protein-1 and macrophage inflammatory protein-1alpha as possible biomarkers for the chronic pelvic pain syndrome. J Urol 2008; 179:1857-61; discussion 1861-2.

11. Zhang N, Inan S, Cowan A, Sun R, Wang JM, Rogers TJ, et al. A proinflammatory chemokine, CCL3, sensitizes the heat- and capsaicin-gated ion channel TRPV1. Proc Natl Acad Sci U S A 2005;102:4536-41.

12. Yang CC, Lee JC, Kromm BG, Ciol MA, Berger R. Pain sensitization in male chronic pelvic pain syndrome: why are symptoms so difficult to treat? J Urol 2003;170:823-6; discussion 826-7.

13. Yilmaz U, Liu YW, Berger RE, Yang CC. Autonomic nervous system changes in men with chronic pelvic pain syndrome. J Urol 2007;177:2170-4; discussion 2174.

14. Sator-Katzenschlager SM, Scharbert G, Kress HG, Frickey N, Ellend A, Gleiss A, et al. Chronic pelvic pain treated with gabapentin and amitriptyline: a randomized controlled pilot study. Wien Klin Wochenschr 2005;117:761-8.

15. Lewis SC, Bhattacharya S, Wu O, Vincent K, Jack SA, Critchley $\mathrm{HO}$, et al. Gabapentin for the management of chronic pelvic pain in women (GaPP1): a pilot randomised controlled trial. PLoS One 2016;11:e0153037.

16. Dworkin RH, Corbin AE, Young JP Jr, Sharma U, LaMoreaux L, Bockbrader $\mathrm{H}$, et al. Pregabalin for the treatment of postherpetic neuralgia: a randomized, placebo-controlled trial. Neurology 2003;60:1274-83.

17. Pontari MA, Krieger JN, Litwin MS, White PC, Anderson RU, McNaughton-Collins $M$, et al.; Chronic Prostatitis Collaborative Research Network-2. Pregabalin for the treatment of men with chronic prostatitis/chronic pelvic pain syndrome: a randomized controlled trial. Arch Intern Med 2010;170:158693.

18. Taylor CP. The biology and pharmacology of calcium channel alpha2-delta proteins Pfizer Satellite Symposium to the 2003 Society for Neuroscience Meeting. Sheraton New Orleans Hotel, New Orleans, LA November 10, 2003. CNS Drug Rev 2004;10:183-8.

19. Devi P, Madhu K, Ganapathy B, Sarma G, John L, Kulkarni C. Evaluation of efficacy and safety of gabapentin, duloxetine, and pregabalin in patients with painful diabetic peripheral neuropathy. Indian J Pharmacol 2012;44:51-6.

20. Toth C. Substitution of gabapentin therapy with pregabalin therapy in neuropathic pain due to peripheral neuropathy. Pain Med 2010;11:456-65.

21. Clark K, Quinn SJ, Doogue M, Sanderson C, Lovell M, Currow DC. Routine prescribing of gabapentin or pregabalin in supportive and palliative care: what are the comparative performances of the medications in a palliative care population? Support Care Cancer 2015;23:2517-20.

22. Agarwal MM, Elsi Sy M. Gabapentenoids in pain management in urological chronic pelvic pain syndrome: gabapentin or pregabalin? Neurourol Urodyn 2017;36:2028-33. 